Square one world media

URL: <http://www.squareoneworldmedia.com/ministries/low-german/ekj-ran/>, letzter Zu-

Statistics Canada griff: 25.07 .2017 .

URL: <https://www12.statcan.gc.ca/nhs-enm/2011/as-sa/99-010-x/99-010-x2011001-eng. cfm\#a3>, letzter Zugriff: 25.07.2017.

Steinbachonline.com

URL: <https://steinbachonline.com/local/mennonitische-post-celebrating>, letzter Zugriff: 25.07.2017.

Thompson, Chad

1994 The Languages of the Amish of Allen County, Indiana: Multilingualism and Convergence. Anthropological Linguistics 36. 69-91.

United States Census Bureau

URL: $<$ https://factfinder.census.gov/faces/tableservices/jsf/pages/productview.xhtml?pid=ACS

Veith, Werner

16_1YR_B04006\&prodType=table>, letzter Zugriff: 03.05.2018.

1968 Pennsylvaniadeutsch: Ein Beitrag zur Entstehung von Siedlungsmundarten. Zeitschrift für Mundartforschung 35. 254-283.

Wikipedia

Wildfeuer, Alfred

URL: <https://en.wikipedia.org/wiki/Old_Order_Mennonite>, letzter Zugriff: 25.07.2017.

2017 Sprachenkontakt, Mehrsprachigkeit und Sprachverlust. Deutschböhmisch-bairische Minderheitensprachen in den USA und in Neuseeland (Linguistik - Impulse \& Tendenzen 73). Berlin \& New York: De Gruyter Mouton.

Wilkerson, Miranda E. \& Joseph Salmons

2008 "Good Old Immigrants from Yesteryear" Who Didn't Learn English: Germans in Wisconsin. American Speech 83. 259-283.

World Alive

URL: <https://www.wycliffe.ca/wycliffe/ck_assets/admin/files/wam/wam_2008_fall.pdf>, letzter Zugriff: 25.07.2017.

Mark L. Louden, Wisconsin (USA)

\title{
45. Deutsch als Minderheitensprache in Mittel- und Südamerika
}

1. Vorbemerkungen

2. Gesamtsituation der komplexen Überdachung: Die multilinguale Situation

3. Auswahl einer prototypischen Situation und Beschreibung des sprachlichen Repertoires
4. Sprachpolitische und soziolinguistische Aspekte, sprachliche Vitalität

5. Deutsch als Fremdsprache in Mittel- und

Südamerika mit einem Fokus auf Brasilien

6. Ausblick

7. Literatur

\section{Vorbemerkungen}

Aus der Vielzahl an deutschsprachigen Siedlungen (in denen Deutsch den Status einer Minderheits-, jedoch keiner staatlichen Amtssprache innehat, siehe Ammon 2015: 299) 
in Mittelamerika (Belize, Puerto Rico) und Südamerika (Argentinien, Bolivien, Brasilien, Chile, Ecuador, Paraguay, Peru, Uruguay, Venezuela) findet eine Beschränkung auf folgende statt: Mennoniten (Belize/Mittelamerika), Hunsrücker (Brasilien/Südamerika) und Deutschböhmen (Brasilien/Südamerika). Die Wahl dieser drei Gruppen betrachten die Verfasser als sinnvoll, da damit sowohl nieder- und mittel- als auch oberdeutsche Varietäten Berücksichtigung finden und neben einem Schwerpunkt auf Südamerika auch der mittelamerikanische Raum thematisiert wird. Es werden sowohl „,religiös konstituiert[e] deutsch(sprachig)[e] Minderheiten“" (Ammon 2015: 369) als auch nicht-religiöse thematisiert. Zunächst werden die historischen und aktuellen Bedingungen dreier Minderheitensprachen in Belize und Brasilien geschildert. In Kap. 2 wird exemplarisch und prototypisch die sprachliche Situation der oberdeutschen Minderheitensprache in Brasilien vorgestellt, bevor im nächsten Punkt soziolinguistische und soziopolitische Faktoren erörtert werden. Die Beschreibung der Deutsch-als-Fremdsprache-Situation in Brasilien schließt den Beitrag.

\section{Gesamtsituation der komplexen Überdachung: Die multilinguale Situation}

\subsection{Deutsch in Mittelamerika - Mennoniten in Belize}

Mennoniten gehören (neben den Amischen und den Hutterern) zu deutschsprachigen Minderheiten, die sich in erster Linie durch religiöse Zusammengehörigkeit identifizieren. Es handelt sich jeweils um Täuferbewegungen, die eine Erwachsenentaufe praktizieren und die ihren Ursprung in den protestantischen Reformationsbewegungen in Europa haben, einen Pazifismus praktizieren und in relativer Abgeschlossenheit leben (siehe Ammon 2015: 381). Die Mennoniten sind in zwei Hauptgruppen zu differenzieren: Einerseits die Pennsylvania German (eine mitteldeutsche Varietät) sprechenden „Schweizer Brüder“, woraus auch die Amischen hervorgegangen sind, und andererseits die „Russland-Mennoniten“, die Plautdietsch (eine niederdeutsche Varietät) sprechen. Auf Letztere konzentrieren sich die folgenden Ausführungen (siehe Ammon 2015: 381). Die mennonitische Historie ist durch zahlreiche Migrationsbewegungen gekennzeichnet. Um 1540 verließen Mennoniten das niederländische Flandern und Friesland und zogen ins Weichsel-Nogat-Delta, von wo sich 900 Mennoniten 1788 nach Russland aufmachten, um dort ein Jahr später die erste Kolonie namens Chortitza zu errichten (siehe Ammon 2015: 382; Steffen 2006: 16). Chortitza wird auch als "Alte Kolonie“" bezeichnet, in Abgrenzung zur „Neuen Kolonie“ in Molotschna, deren Gründung auf 1804 datiert werden kann (siehe Steffen 2006: 17 u. 29). Als die Regierung Russisch als Unterrichtssprache in den mennonitischen Schulen und Militärdienst forderte, wanderten ab 1873 konservative Mennoniten nach Nordamerika aus (siehe Kaufmann 1997: 59; Ammon 2015: 382). Die Belizer Mennoniten ließen sich nach der Emigration aus Kanada zunächst in Mexiko nieder (vor allem in Chihuahua und Durango) und wanderten dann nach Belize aus (siehe Steffen 2006: 19). Belize wurde bis 1973 als „British Honduras" bezeichnet, 1981 wurde der Staat unabhängig (siehe Ammon 2015: 383). 1957 wurde zwischen den Mennoniten und der damaligen britischen Kolonie Honduras ein Vertrag geschlossen, der Sondergenehmigungen in Bezug auf die soziale, politische 


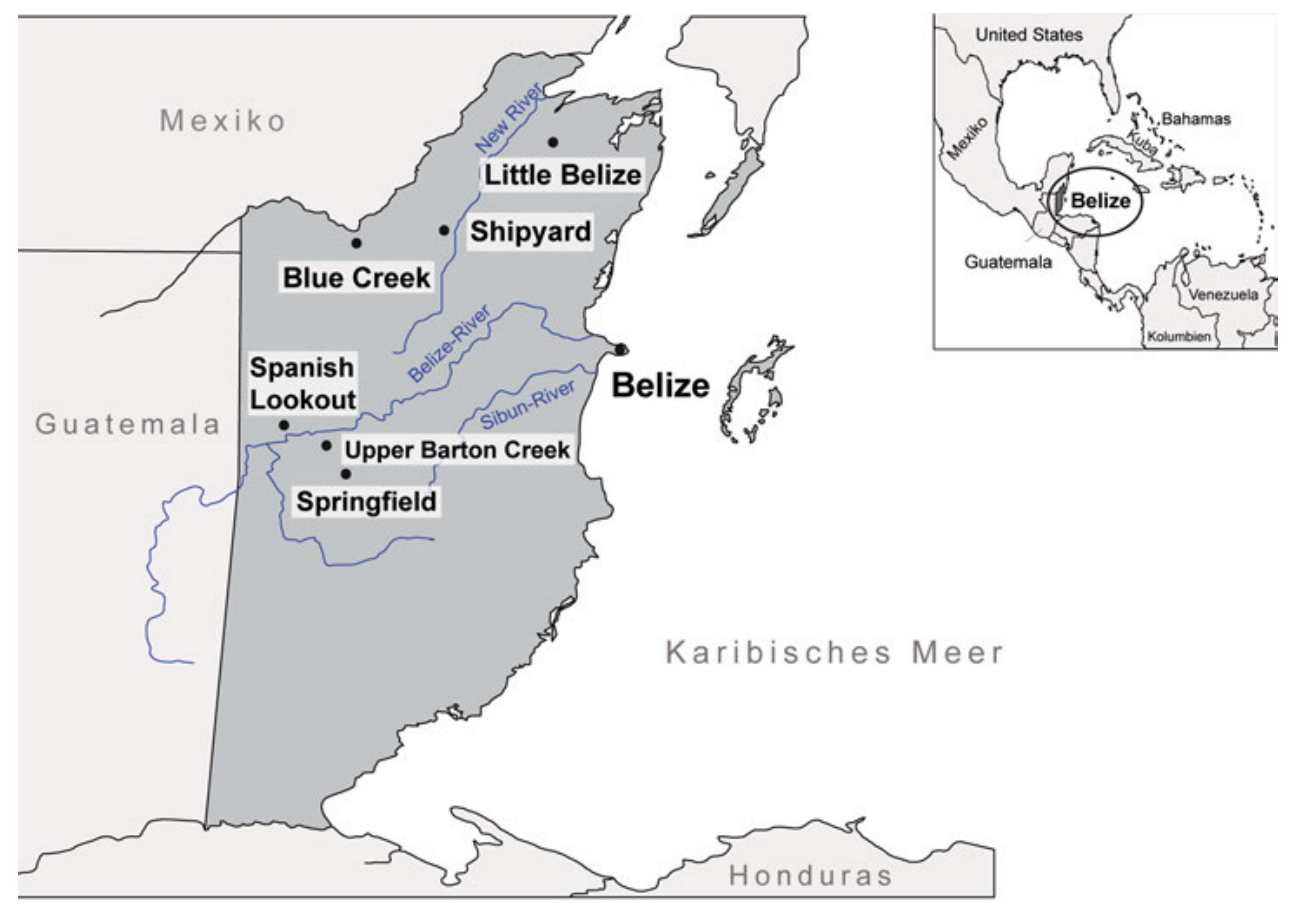

Abb. 45.1: Siedlungsgebiete der Mennoniten in Belize

und wirtschaftliche Situation enthielt (siehe Steffen 2006: 20). Bis zum Jahr 1966 kamen circa 200 Familien und insgesamt 2.700 Personen nach Belize (siehe Steffen 2006: 21). Im aktuellen Ethnologue (2016) wird eine Zahl von 9.360 Plautdietschsprechenden für den Cayo- und den Orange Walk-Distrikt (die im Westen und Nordwesten des Landes liegen und in denen sich die betreffenden Mennonitenkolonien befinden) genannt, der sprachliche Status der Sprechergemeinschaft wird als „,vigorous“ ('lebhaft, vital') bezeichnet.

Ammon (2015: 302) klassifiziert die Mennoniten in Belize als eine deutschsprachige Minderheit mit religiöser Identität, die in insgesamt sechs Kolonien differenziert werden kann (vgl. Abb. 45.1: Little Belize, Shipyard, Blue Creek, Spanish Lookout, Upper Barton Creek und Springfield) und die sich hinsichtlich ihrer wirtschaftlichen Interessen, der sprachlichen Situation und der kulturellen Ausprägung deutlich unterscheiden (siehe Steffen 2006: 174).

Diese Unterschiede werden nachfolgend anhand zweier konträrer Gruppierungen (Blue Creek und Shipyard) verdeutlicht.

Die ersten Mennoniten siedelten ab 1958 in Shipyard. Sie sind zu den Altkoloniern zu zählen, die zu den „konservativsten mennonitischen Gruppen“ gehören, explizit den Kontakt zur Außenwelt gering halten und als Transport- und Fortbewegungsmittel nach wie vor die Pferdekutsche verwenden, „da ihnen das Fahren von motorisierten Autos oder Lastkraftwagen untersagt ist" (Steffen 2006: 29). Dennoch sind die Altkolonier auf „Gewinnmaximierung“ ausgerichtet (Steffen 2006: 172). 
Blue Creek wurde ebenfalls 1958 von mennonitischen Auswanderern aus dem mexikanischen Chihuahua etabliert, war zu Beginn ebenfalls altkolonischer Glaubensrichtung und verfügte ,zunächst [über] die gleiche Gesellschaftsordnung wie Shipyard und Little Belize und die gleichen Sitten und Gebräuche [...]" (Steffen 2006: 32). Aufgrund von Differenzen über von einigen Siedlern mitgebrachte motorisierte Hilfsmittel kam es zur Separation innerhalb der Gemeinde, was dazu führte, dass die konservativen Altkolonier entweder nach Shipyard zogen oder nach Bolivien emigrierten. In Blue Creek entstand dann 1966 die Gemeinschaft der „Evangelical Mennonite Mission Conference“ (Steffen 2006: 33). Befreit von den Zwängen der Altkolonier kam es zu einer anhaltenden wirtschaftlichen Prosperität, die aber keineswegs eine vollständige Assimilation der Mennoniten nach sich zog. Ein Effekt der Öffnung der Kolonie ist sicherlich auch, dass der Besitz und Gebrauch von TV-Geräten, Radios, Computern und dem Internet erlaubt sind (siehe Steffen 2006: 33-34).

Die präsentierten Kolonien teilt Steffen in differierende „Gesellschaftstypen“ ein: Die Altkolonier in Shipyard tituliert er als ,societés froides“, die ihr Augenmerk ,auf die Bewahrung der gegenwärtigen Verhältnisse“ richten (Steffen 2006: 175). Im Gegensatz dazu findet man in Blue Creek die ,societé chaude“, in der „die wirtschaftliche und gesellschaftliche Veränderung und Entwicklung" durchaus intendiert ist und intensiviert wird (Steffen 2006: 175-176).

Die beiden Gesellschaftstypen verhalten sich auch hinsichtlich ihrer ,,polyglossischen Situation“ (Steffen 2006: 173) unterschiedlich: In Blue Creek ist in der älteren Generation Plautdietsch nach wie vor die Umgangssprache. Als Schriftsprache wird ,eine archaische Form“ des Hochdeutschen verwendet (Steffen 2006: 24), die als Sprache des Gottesdienstes jedoch längst vom Plautdietschen verdrängt wurde, „da die hochdeutsche Sprache nicht mehr als integraler Bestandteil der Religion gesehen wird“" (Steffen 2006: 176). In der jungen Generation fungiert neben dem Plautdietschen bereits das Englische als Umgangssprache. Als Schulsprache wurde das Hochdeutsche bereits durch das Englische substituiert.

In Shipyard hingegen ist die Domänenverteilung Plautdietsch als Low- und Hochdeutsch als High-Varietät in der älteren Generation wohl konstant. Dies wird durch den Sachverhalt gestützt, dass es für viele „die einzige Sprache ist, die sie beherrschen“ (Steffen 2006: 177). Allerdings befindet sich das Plautdietsche durch den Sprachkontakt mit dem Spanischen in einem Entlehnungsprozess, der zu sprachlichen Innovationen führt (siehe Steffen 2006: 177). Schulsprache ist nach wie vor das Hochdeutsche, ,welches den Schülern allerdings wie eine Fremdsprache vorkommen muß, da sie bis zum Schulalter kaum Kontakt mit der Sprache haben“ (Steffen 2006: 88). Der überwiegende Teil der jüngeren Generation verfügt über eingeschränkte Kenntnisse im Hochdeutschen, weshalb dieses als „Dummy High, d. h. als dysfunktionale Hochsprache, gekennzeichnet“ ist (Steffen 2006: 178).

Die kontaktsprachliche Situation in Belize gestaltet sich aufgrund des Neben- und Miteinanders heterogener Bevölkerungsgruppen komplex (siehe Steffen 2006: 1). Belize ist das einzige Land Südamerikas, in dem Englisch die offizielle Landessprache ist. Daneben existieren laut Ethnologue (2016) Belize Kriol Englisch, das von Steffen (2006: 94) als die ,inoffizielle Landessprache Belizes“ bezeichnet wird, drei indigene Sprachen (Kekchí, Mopán Maya, Yucatec Maya), Spanisch und Garifuna, das auch als „Black Carib" bezeichnet wird. 
Die für die vorgestellten mennonitischen Kolonien relevanten Kontaktsprachen sind Englisch, Spanisch und das Belize Kriol (siehe Steffen 2006: 180). Der Einfluss der englischen Sprache, obwohl es die einzige offizielle Landessprache ist, ist in den Mennonitenkolonien durchaus divergent. Während in Blue Creek das Englische bereits als Schul- und teilweise auch als Gottesdienstsprache verwendet wird, hat es in der Altkoloniersiedlung Shipyard nur marginale Bedeutung. Gegenüber dem Spanischen herrscht in den Kolonien durchaus eine „utilitaristische Werthaltung“ (Steffen 2006: 181), wobei hinsichtlich der Sprachkompetenz durchaus genderspezifische Unterschiede vorhanden sind. In Shipyard beispielsweise ist Sprachkompetenz im Spanischen in erster Linie bei den Männern existent (siehe Steffen 2006: 92).

In den Belizer Mennonitenkolonien ist das Plautdietsche nach wie vor zweifelslos die „gemeindeübergreifende Umgangssprache“, die sich in den einzelnen Kolonien jedoch unterschiedlich entwickelt, da „,das Spanische stärkere Spuren in den nördlichen Kolonien hinterläßt, während der Einfluß des Englischen deutlicher in den zentralbelizischen Kolonien festzustellen ist [...]“ (Steffen 2006: 182).

\subsection{Deutsch in Südamerika - Brasilien}

\subsubsection{Hunsrücker}

Das „brasilianisch[e] Kolonisationsgesetz“ von 1820 sowie die Verfassung von 1824 ermöglichten die (auch nicht-katholische) Einwanderung aus deutschsprachigen Gebieten, und es wurden ausgehend von São Leopoldo Regionen der südbrasilianischen Staaten Rio Grande do Sul, Santa Catarina, Paraná und Espírito Santo bevölkert (Ammon 2015: 369). Die ersten Gründungen werden als die „Alten Kolonien“ bezeichnet, woraus Tochterkolonien bzw. -gründungen hervorgingen (siehe Altenhofen 2016: 106 u. 108). Um einen raschen Sprachwechsel zum Portugiesischen zu bewirken, fokussierte die Regierung ,eine Politik der ethnisch gemischten Ansiedlung“ (Ammon 2015: 369; siehe dazu auch Altenhofen 1996: 68-69). Heutzutage kann man davon ausgehen, dass in Brasilien circa zwei bis fünf Millionen Deutschstämmige, darunter 600.000 bis 1.500.000 deutschsprechende Personen anzutreffen sind (siehe Grabarek 2013: 206; Ammon 2015: 373). Ammon (2015: 373) erwähnt allerdings, dass derartige Zahlenangaben generell mit Vorsicht zu behandeln sind, da die letzte verlässliche Volksbefragung auf $1950 \mathrm{zu}$ datieren ist. Als dominierende deutschsprachige Gruppe innerhalb Brasiliens gelten zweifelslos die Hunsrücker. Neben diesen existieren auch noch ,schwäbischalemannische, bairisch-österreichische, westfälische und pommerische" Sprechergruppen (Ammon 2015: 371). Ammon (2015: 371) verweist darauf, dass „Hunsrücker“ (auch „Riograndenser Hunsrücker“) von ihm als „Pars pro toto“-Bezeichnung für alle „autochthonen Deutsch(sprachig)en in Südbrasilien“ verwendet wird.

Das Hunsrückische ist als „Koine“ zu charakterisieren (Altenhofen 1996: 5, 2016: 104), ,deren dialektale Merkmale auf die rhein- und moselfränkische Basis der deutschen Urheimat zurückzuführen sind.“ Der Terminus „Hunsrücker“ wird in Rio Grande do Sul „delokutiv durch dritte Personen“ verwendet (Altenhofen 1996: 5). Der Hunsrücker hingegen benennt sich selbst als „Daitscher“ (Altenhofen 1996: 5). In den Alten Kolonien Rio Grande do Suls allerdings fungiert der Terminus „Hunsrückisch“ (oder auch „Hunsbucklisch“) noch bisweilen als Selbstbezeichnung (siehe Altenhofen 2016: 116). 


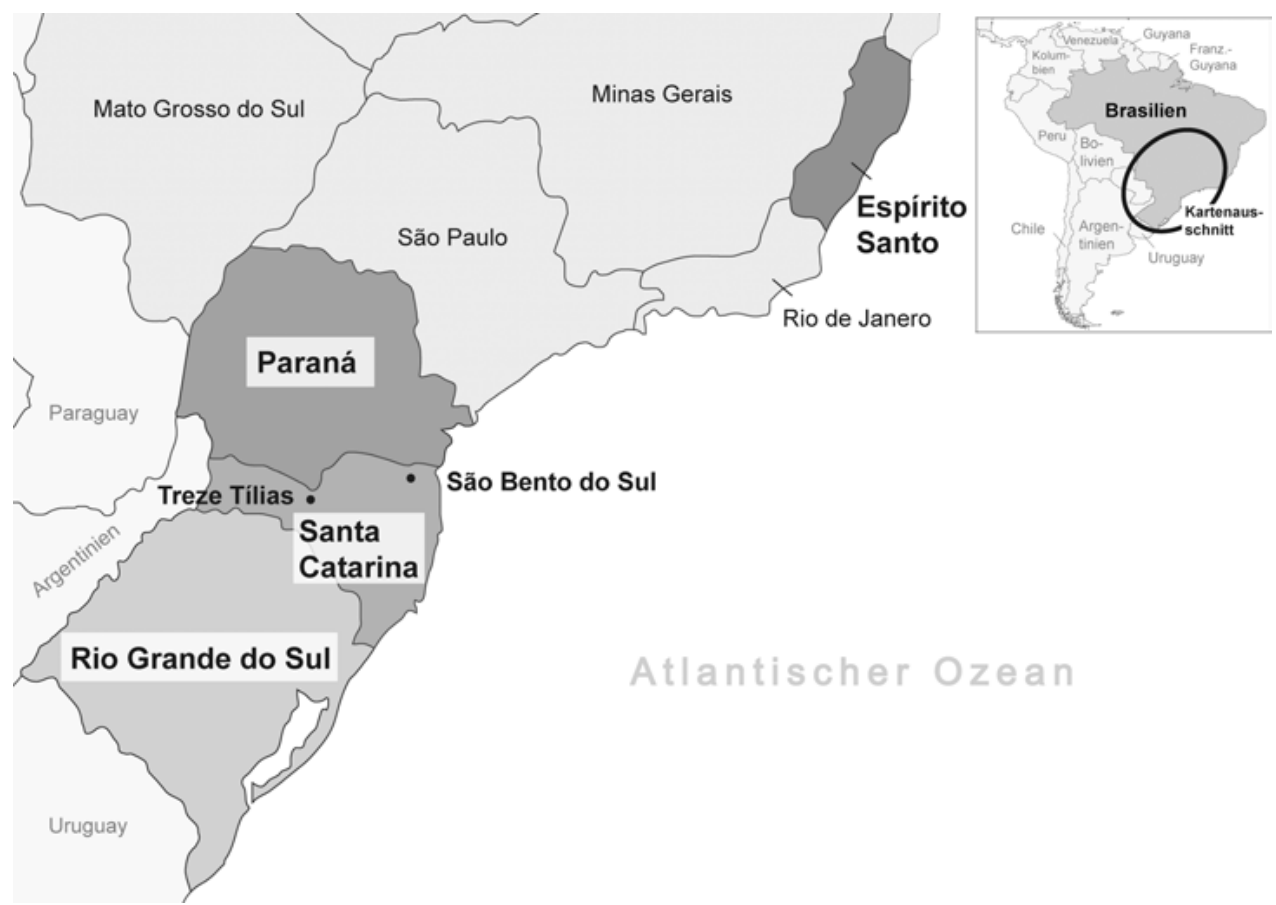

Abb. 45.2: Siedlungsgebiete der Hunsrücker in Südbrasilien

Das hunsrückische Siedlungsgebiet konzentriert sich in Brasilien in erster Linie auf die bereits erwähnten Bundesstaaten Rio Grande do Sul, Santa Catarina, Paraná und Espírito Santo (siehe Ammon 2015: 372) und ist auf Abb. 45.2 ersichtlich.

Die Mehrheit der Hunsrücker lebt zentriert in Dörfern, die jedoch kein konformes Siedlungsgebiet bilden (siehe Ammon 2015: 373). Allerdings sollten die als „Kolonien“ klassifizierten Siedlungsgebiete und -gemeinschaften Uniformität suggerieren (Ammon 2015: 373). Das hunsrückische Sprachgebiet ist vielmehr durch Verstreutheit (siehe Steffen \& Altenhofen 2014: 35) gekennzeichnet, was die Entstehung von „Fami(lio)lekten" bewirkte, die dann auch die Grundlage für Sprachausgleich bildeten (Altenhofen 1996: 345). Es kann daher von einem „Archipel“ gesprochen werden, welcher „durch identische Strukturen und Bräuche sowie eine gemeinsame Sprache zusammengehalten“ wird (Steffen \& Altenhofen 2014: 56). Ein Merkmal des Hunsrückischen ist, dass es einen enormen sprachlichen Unterschied zum Standarddeutschen aufweist und dass es sich um eine deutschbasierte Minderheitensprache handelt (siehe Steffen \& Altenhofen 2014: 37). Steffen \& Altenhofen sprechen daher auch von der ,Souveränität des Dialekts“, von einer „Prävalenz der Mündlichkeit“" und von einer ,,doppelten Alietät“ (Steffen \& Altenhofen 2014: 37), einer doppelten Abgrenzung sozusagen, was bedeutet, dass Hunsrückisch sich sowohl von der sprachlichen Umgebung in Brasilien unterscheidet als auch von den Dialekten in Deutschland, aus denen es hervorgegangen ist. Der erwähnten „Prävalenz der Mündlichkeit“ ist allerdings entgegenzusetzen, dass im Projekt ESCRITHU basierend auf dem Standarddeutschen Regeln zur Verschriftlichung des 
Hunsrückischen entwickelt wurden. ESCRITHU (siehe Pupp Spinassé 2016: 88) ist ein Teilprojekt des Großprojekts ALMA-H (Atlas Linguístico-Contatual das Minorias Alemãs na Bacia do Prata - Hunsrückisch). Als Beispiel für die Verschriftlichung des Hunsrückischen im wissenschaftlichen Kontext dient die Hunsrickisch Red fo die Sprocherechte von Altenhofen \& Frey (2006).

Die multilinguale Situation, in der die Hunsrücker sich sprachlich bewegen, ist komplex und wird von Altenhofen (1996: 26) als „Varietätennetzwerk“ bezeichnet, welches durch mindestens acht Kontaktkonstellationen determiniert ist. Diese Konstellationen sind nach Altenhofen (1996: 26) die mosel- und rheinfränkische Varietätengrundlage, das Latein als wohl primäre Kontaktsprache, das Französische (womit die Varietäten im Ursprungsland in Kontakt standen), die portugiesische Varietät in Rio Grande do Sul, eine Ausprägung des ,lokale[n] Hochdeutsch“, weitere Varietäten des Deutschen, die in der Umgebung gesprochen werden (wie zum Beispiel Schwäbisch, DeutschböhmischBairisch, Westfälisch), weitere Einwanderersprachen (wie Polnisch, Italienisch) und zuletzt auch die Kontaktsprachen der indigenen Bevölkerung, wenngleich deren Einfluss sicherlich marginal ist.

Ziegler beschreibt die sprachliche Situation, in der ein prototypischer Hunsrücker sich befindet, als „soziales Kontinuum“ mit den jeweiligen Polen Hunsrücker Varietät und portugiesische Standardsprache (beziehungsweise eine Ausprägung davon) (Ziegler 1996: 83). In der Mitte dieses Gefüges befindet sich das so genannte „Misturado“, welches die Funktion einer Umgangssprache innehat und durch eine Vielzahl an ,portugiesischen Interferenzen“" gekennzeichnet ist (siehe Ziegler 1996: 73 u. 83). Ein anschauliches Beispiel hierfür ist: „De Man hat de mulo mit de rehle durch de banhado in das potrea ketockt. (Der Mann hat den Esel (portug. a mula) mit der Peitsche (portug. o relho) durch den Sumpf (portug. o banhado) auf die Weide (portug. o potreiro) getrieben (portug. tocar))“" (Ziegler 1996: 73).

In Bezug auf das Sprachenrepertoire erwähnt Pupp Spinassé (2016: 96), dass zahlreiche ältere Personen Defizite im Portugiesischen hätten bzw. eine deutliche Präferenz vorläge, auch bei ausgeprägter Sprachkompetenz im Portugiesischen, Hunsrückisch zu kommunizieren. Ziegler geht zudem davon aus, dass die Standardvarietät zwar von nahezu allen Hunsrückern verstanden wird, jedoch nicht fehlerfrei geschrieben bzw. gesprochen werden kann (siehe Ziegler 1996: 47).

Altenhofen charakterisiert die Domänenverwendung des Portugiesischen und der Hunsrücker Varietät folgendermaßen: „Das Ptg. [Portugiesische] wurde zum Symbol der Stadt, der höheren Schicht, des Wissens, der Schule, der Nationalität und der jüngeren Generation. Das Hrs. [Hunsrückische] wird im Gegensatz dazu wachsend mit der Sprache der ländlichen Gegenden, der Herkunft, der Familie, der Gruppensolidarität und der älteren Generation assoziiert.“ (Altenhofen 1996: 73).

Die beschriebene Domänenteilung wird ebenfalls von Ammon (2015: 376) erwähnt, der anführt, dass keine verlässlichen Daten vorliegen, wie viele Kinder heutzutage noch Hunsrückisch als Primärsprache erlernen (siehe Ammon 2015: 374). Dass die Familie für den Erhalt des Hunsrückischen vor allem in den ländlichen Regionen als eine Art „sprachliches Refugium“ fungiert, attestiert Altenhofen (2016: 126) auch für die rezenten Verhältnisse. Allerdings konnte in Interviewsituationen, die auf Hunsrückisch abliefen, beobachtet werden, dass Erwachsene mit Kindern und Jugendlichen auf Portugiesisch kommunizieren (siehe Altenhofen 2016: 126). 


\subsubsection{Deutschböhmen}

„Deutschböhmen“ ist ein Terminus, der impliziert, dass die entsprechenden Personen deutsch(sprachig)er Abstammung waren und in einem Territorium siedelten, welches zum heutigen Westtschechien gehört. Nachfahren dieser Deutschböhmen, die eine oberdeutsche Varietät (genauer einen nordmittelbairischen Mischdialekt) sprechen, leben noch heute im südbrasilianischen Bundesstaat Santa Catarina, vor allem in der Stadt São Bento do Sul (zur weltweiten Verbreitung dieser Gruppe siehe Wildfeuer 2016). Da die Herkunftsorte der bairischen Ansiedler von Saõ Bento diesseits und jenseits der bayerischen Ostgrenze lagen (siehe Blau 1958: 10), meint Deutschböhmen in diesem besonderen Falle auch einige Personen bayerischer Abstammung. Die Siedlungsgeschichte der so genannten Deutschböhmen beschreibt Blau (1958: 7-8) wie folgt: „Erst 1873 begann die Hamburger Ansiedlungsgesellschaft mit der Besiedlung des Hochlandes von Sao [sic!] Bento, die die ersten Kolonisten baierischen Volkstums nach Südamerika brachte; [...] die nächste baierische Ansiedlung Dreizehnlinden [Treze Tílias] unter dem österreichischen Minister Thaler kam erst 1933, [...].“ 1873 machten sich 26 Deutschböhmen auf den Weg nach Brasilien (siehe Blau 1958: 17), 1876 kamen weitere 300 Auswanderer aus Bayern und dem Böhmerwald hinzu (siehe Blau 1958: 96). Einer Ansiedlungsliste São Bentos für die Jahre 1874-1878 nach betrug der Anteil von Personen aus dem Böhmerwald und Bayern insgesamt 225, was einem Prozentsatz von 46,2\% entspricht. Die Deutschböhmen und ihre Varietät waren von Anfang an im Kontakt mit weiteren bairischen und norddeutschen Varietäten, dem Pommerischen, dem Polnischen, dem Brasilianischen, dem Französischen und auch dem Tschechischen (siehe Blau 1958: 46).

Mit Beginn des Ersten Weltkriegs wurden 1914 alle Schulen mit deutscher Schulsprache unverzüglich geschlossen, und es entstanden Schulen mit portugiesischer Unterrichtssprache. Diese konnten anfänglich jedoch nur geringe Akzeptanz verzeichnen. Hingegen war die früher praktizierte Variante, Portugiesisch in deutschen Schulen zu unterrichten, erfolgreicher, da damit eine sprachliche Basis gelegt worden war, die im alltäglichen Umgang mit Portugiesisch Sprechenden dann vertieft werden konnte (siehe Blau 1958: 101).

Ab 1938 verschärfte sich die Lage zunehmend, und es kam zu einer Verbannung der deutschen Sprache aus dem öffentlichen Leben, die laut Blau auch zur Folge hatte, dass das Deutsche nicht mehr an Jüngere tradiert wurde (siehe Blau 1958: 105).

Ganz so hoffnungslos zeigt sich die Lage aktuell nicht. Von den Verfassern durchgeführte Interviews mit deutschböhmischen Nachfahren aus Saõ Bento stellen unter Beweis, dass es durchaus noch zahlreiche kompetente Sprecher auch innerhalb der mittleren Generation gibt. Die Zahl kann laut Aussage von Informanten alleine in Saõ Bento auf ca. 700 bis 1.000 geschätzt werden, und es scheint wohl auch so zu sein, dass es noch einige wenige jüngere Sprecherinnen und Sprecher gibt (siehe hierzu auch EllerWildfeuer 2016). 2007 und 2011 führten die Verfasser mit insgesamt drei Informanten, die die Herkunftsorte ihrer Vorfahren in Tschechien besuchten, Befragungen in Form von sprecherbiographischen Interviews durch.

Das sprachliche Repertoire der interviewten Personen besteht aus einer Ausprägung der deutschen Varietät und der portugiesischen Sprache. Ihre deutsche Varietät bezeichnen sie als „Bairisch" oder auch „Boarisch“ und sie sind stolz darauf, diese noch zu schnattern ('sprechen').

Bei den Befragten fand, mit Ausnahme einer Informantin, die mit ihrem Mann nach wie vor Deutschböhmisch spricht und die Varietät auch an ihre Kinder tradierte, eine 
Abkehr von der einstigen Primärsprache hin zum Portugiesischen statt. Die drei Befragten verfügen nach wie vor über ausgeprägte Kompetenzen im Deutschböhmischen. An ihre Kinder haben zwei der drei Befragten die Varietät nicht tradiert. Ein Sohn, der bei der Befragung 2007 anwesend war, verfügt lediglich über passive Sprachkompetenz.

\section{Auswahl einer prototypischen Situation und Beschreibung des sprachlichen Repertoires}

Im Folgenden wird anhand der deutschbasierten Minderheitensprache der Deutschböhmen in São Bento (Santa Catarina) die sprachliche Situation konzise dargestellt.

Exemplarisch wird ein Ausschnitt eines Interviews, das im September 2007 durchgeführt wurde, transkribiert wiedergegeben. Der befragte Sprecher A. B. wurde 1949 in São Bento in eine deutschböhmische Familie hineingeboren und wohnt bis heute dort. Seine Vorfahren sind nach seiner Auskunft um 1870 aus dem östlich des Künischen Gebirges (Královský Hvozd) liegenden Hammern/Hamry (Okres Klatovy, Tschechien) nach Brasilien ausgewandert. Er benutzt nach eigenen Angaben die deutschböhmische Varietät noch gelegentlich in der Kommunikation mit seiner Ehefrau und mit Geschwistern. Seine Kinder dagegen haben die Minderheitensprache nicht mehr erworben. Folgende Ausschnitte aus dem etwa halbstündigen Interview geben einen Einblick in die deutschböhmische Minderheitensprache und in die aktuelle Situation der Sprachverwendung vor Ort. Auf eine enge phonetische Transkiption wird an dieser Stelle verzichtet. Das Interview wurde mit dem Transkriptionstool EXMARaLDA transkribiert und annotiert (die Abkürzung A. B. steht für die Gewährsperson, N. E. für Nicole Eller-Wildfeuer, A. W. für Alfred Wildfeuer, K. für Kommentare und Ü. für eine Übertragung ins Standarddeutsche). Einzelne für den Argumentationsgang relevante Belege werden nach den Konventionen von IPA transkribiert.

\subsection{Transkription eines freien Gespräches mit Gewährsperson A. B.}

$[1]$

\begin{tabular}{lll}
\hline & $1[03: 04.1]$ & $2[03: 07.3]$ \\
\hline A. W. $[\mathbf{v}]$ & Und ähm iats vo enk enkane kinna & $(--)$ kinna de $(-)$ no Boarisch oda wochsn de dann \\
\hline Ü. $[\mathbf{v}]$ & Und ähm jetzt von euch, euere Kinder, & können die noch Bairisch oder wachsen die dann \\
\hline
\end{tabular}

\begin{tabular}{lll}
\hline & $\ldots$ & $3[03: 12.5]$ \\
\hline A. $\mathbf{B} .[\mathbf{v}]$ & & $(1,9)$ mia $[\ldots]$ hätt mas ea a so ament sogoa \\
\hline A. W. $[\mathbf{v}]$ & $($.$) nur no mit Portugiesisch af$ & \\
\hline$\ddot{\mathbf{U}}[\mathbf{~} \mathbf{v}]$ & nur noch mit Portugiesisch auf? & Wir hätten es ihnen so vielleicht sogar \\
\hline K. $[\mathbf{k}]$ & & anfänglich Geräusche im Hintergrund, Teile schwer verständlich \\
\hline
\end{tabular}


[3]

\begin{tabular}{llll}
\hline & $4[03: 16.6]$ & $5[03: 17.5]$ & $6[03: 19.3]$ \\
\hline A. B. $[\mathbf{v}]$ & $($.$) glernd und homa$ & homas ea willn lerna & $(--)$ owas schlecht $($.$) is$ \\
\hline Ü. $[\mathbf{v}]$ & gelernt und haben, & haben es ihnen wollen lernen, & aber das Schlechte ist, \\
\hline
\end{tabular}

[4]

\begin{tabular}{lllll}
\hline & 7 [03:20.7] & $8[03: 21.9]$ & $9[03: 23.7]$ & $10[03: 25.0]$ \\
\hline A. B. $[\mathbf{v}]$ & waa (.) d nachbarn & $(--)$ und nachand is d schul & $(---)$ da unterricht & $(--)$ und television \\
\hline Ü. $[\mathbf{v}]$ & weil die Nachbarn & und nachher ist die Schule, & der Unterricht & und Television \\
\hline
\end{tabular}

[5]
$11[03: 26.5]$
$12[03: 29.3]$

A. B. [v] (.) und alles wei ma song (.) des is alles af Portugiesisch (---) da äh von uns

Ü. [v] und alles, wie wir sagen, das ist alles auf Portugiesisch.

Da äh von uns

[6]
$13[03: 31.8]$
$14[03: 32.8]$

\begin{tabular}{lll}
\hline A. B. $[\mathbf{v}]$ & (.) da älterner hods no a weng gwisst & owa eitzand hod eas scho voglernt \\
\hline Ü. [v] & der Ältere hat es noch ein wenig gewusst, & aber jetzt hat er es schon vergelernt,
\end{tabular}

[7]

\begin{tabular}{llll}
\hline & $15[03: 34.3]$ & $16[03: 34.9]$ & 17 [03:36.1] \\
\hline A. B. $[\mathbf{v}]$ & $(-)$ volernt & wei mia hama a $($.$) i han a$ & $($.$) scho ungefähr zwanzg johr wos ma$ \\
\hline Ü. $[\mathbf{v}]$ & verlernt, & wie wir haben auch, ich habe auch & schon ungefähr zwanzig Jahre, was man \\
\hline K. $[\mathbf{k}]$ & Sprecher verbessert sich. & Sprecher setzt neu an. & \\
\hline
\end{tabular}

[8]

\begin{tabular}{lllll}
\hline & $\ldots$ & $18[03: 38.1]$ & $19[03: 40.3]$ & $20[03: 42.3]$ \\
\hline A. B. $[\mathbf{v}]$ & nimmer & $(--)$ gschnodert hod die Bairische ne & $(---)$ weil die eltern san & $($.$) weggfolln ne$ \\
\cline { 2 - 5 } Ü. $[\mathbf{v}]$ & nicht mehr & geschnattert hat die Bairische, nicht? & Weil die Eltern sind & weggefallen, nicht? \\
\hline
\end{tabular}




\begin{tabular}{llll}
\hline & $21[03: 43.4]$ & $\ldots$ & $22[03: 47.9]$ \\
\hline A. B. $[\mathbf{v}]$ & $(0,9)$ und $(-)$ de meistn hätzadogs schnodern Portugiesisch ne & $(--)$ is schlechd \\
\cline { 2 - 3 } N. E. $[\mathbf{v}]$ & & $\mathrm{mhm}$ \\
\hline Ü. $[\mathbf{v}]$ & Und die meisten heutzutage schnattern Portugiesisch, nicht? & Ist schlecht \\
\hline
\end{tabular}

[10]

\begin{tabular}{|c|c|c|c|c|}
\hline & $\ldots$ & $23[03: 50.0]$ & $24[03: 51.1]$ & $25[03: 53.6]$ \\
\hline A. B. $[\mathbf{v}]$ & zun holtn ne & & & \\
\hline N. E. $[\mathbf{v}]$ & $\mathrm{mhm}$ & $\mathrm{mh}$ & & \\
\hline A. W. $[\mathbf{v}]$ & & $(-)$ ja ja & (---) eitz wenns aber wenns enk es & auf der stroß treffts \\
\hline Ü. [v] & zum halten, nicht? & Ja, ja. & Jetzt wenn ihr, aber wenn euch ihr & auf der Straße trefft, \\
\hline
\end{tabular}

[11]

\begin{tabular}{lll}
\hline & $26[03: 55.0]$ & $27[03: 56.2]$ \\
\hline A. $\mathbf{W} . \mathbf{~} \mathbf{v}]$ & iatz de andern wird dann no & wird do no a weng mh Boarisch gredt oder is dann \\
\hline Ü. $[\mathbf{v}]$ & jetzt, die anderen, wird dann noch, & wird da noch ein wenig Bairisch geredet oder ist dann \\
\hline
\end{tabular}

[12]

\begin{tabular}{lllll} 
& $28[03: 58.2]$ & $29[03: 58.9]$ & $30[04: 00.1]$ & $31[04: 01.4]$ \\
\cline { 2 - 5 } A. B. $[\mathbf{v}]$ & mia rema mia & af a so af da straß & und af de andern \\
\hline A. W. $[\mathbf{v}]$ & eher Portugiesisch & & & \\
\hline Ü. $[\mathbf{v}]$ & eher Portugiesisch? & Wir reden, wir & auf so auf der Straße & und auf die anderen, \\
\hline K. $[\mathbf{k}]$ & & Sprecher setzt neu an. & & \\
\hline
\end{tabular}

[13]

\begin{tabular}{llll}
\hline & $32[04: 02.0]$ & $33[04: 04.0]$ & $34[04: 06.6]$ \\
\hline A. B. $[\mathbf{v}]$ & wenn ma si so begegnd mit epan ne & $(1,1)$ rema fei nur Portugiesisch & nur wenn \\
\hline Ü. $[\mathbf{v}]$ & wenn man sich so begegnet mit jemanden, nicht, & reden wir bloß nur Portugiesisch. & Nur wenn \\
\hline
\end{tabular}

[14]

\begin{tabular}{llll}
\hline & $\ldots$ & $35[04: 08.6]$ & $36[04: 09.8]$ \\
\hline A. B. $[\mathbf{v}]$ & ma mit da famili zamghemand & $(--)$ weil mia samma & $(---)$ acht $($.$) briada$ \\
\hline Ü. $[\mathbf{v}]$ & wir mit der Familie zusammenkommen, & weil wir sind & acht Brüder, \\
\hline
\end{tabular}


$[15]$

\begin{tabular}{llll} 
& 37 [04:11.3] & 38 [04:11.7] & 39 [04:14.3] \\
\hline A. B. $[\mathbf{v}]$ & & (.) san drei deandla (--) und finf boum & $(---)$ wenn ma (.) hinter da famili \\
\hline N. E. $[\mathbf{v}]$ & $\mathrm{mhm}$ & & \\
\hline Ü. $[\mathbf{v}]$ & & sind drei Mädchen und fünf Buben. & Wenn wir unter der Familie \\
\hline
\end{tabular}

[16]

\begin{tabular}{lllll}
\hline & $\ldots$ & $40[04: 17.2]$ & $41[04: 19.1]$ & $42[04: 21.1]$ \\
\hline A. B. $[\mathbf{v}]$ & zamghemand & $(--)$ nahand rema awel & $(---)$ nahand rema (-) deitsch & \\
\hline N. E. $[\mathbf{v}]$ & & & & mhm \\
\hline Ü. [v] & zusammenkommen, & dann reden wir allweil, & dann reden wir Deutsch. & \\
\hline
\end{tabular}

$[17]$

$43[04: 21.7]$ $44[04: 22.5]$

A. B. [v] nahand rema die (--) die sprach no wos ma glernt hamand vo dahoam (.) ne

$\begin{array}{ll}\text { A. W. }[\mathbf{v}] \quad(-) \mathrm{mhm} & \end{array}$

Ü. [v] Dann reden wir die die Sprache noch, was wir gelernt haben von daheim, nicht?

[18]

\begin{tabular}{llll}
\hline & $46[04: 25.7]$ & $47[04: 26.9]$ & $48[04: 27.8]$ \\
\hline A. B. $[\mathbf{v}]$ & weng dem woaß mas no a weng & weil wenn des ned waar & dad mas gor nim (.) \\
\hline Ü. [v] & Wegen dem weiß man es noch ein wenig, & weil wenn das nicht wäre, & täte man es gar nimm-, \\
\cline { 2 - 4 } K. $[\mathbf{k}]$ & & & Sprecher setzt neu an. \\
\hline
\end{tabular}

[19]

\begin{tabular}{|c|c|c|c|}
\hline & $49[04.29 .7]$ & $50[04: 29.9]$ & $51[04: 31.6]$ \\
\hline A. B. $[\mathbf{v}]$ & beinah gor nimma wissn ne & $(1,2)$ waal & $\begin{array}{l}\text { mia sama a grouße famili samma in } \\
\text { acht }\end{array}$ \\
\hline N. E. [v] & $\mathrm{mh}$ & & \\
\hline Ü. [v] & $\begin{array}{l}\text { beinahe gar nicht mehr wissen, } \\
\text { nicht? }\end{array}$ & Weil & $\begin{array}{l}\text { wir sind eine große Familie, sind wir in } \\
\text { acht, }\end{array}$ \\
\hline K. $[\mathbf{k}]$ & & & Sprecher verbessert seine Äußerung. \\
\hline
\end{tabular}


[20]

52 [04:35.5]

53 [04:37.6]

54

[04:40.3]

\begin{tabular}{lllll} 
A. B. $[\mathbf{v}]$ & $(-)$ acht $(-)$ ne & $(-)$ mei älterner bria (.) bruader hod $(1,1)$ seksasechzg johr & und mei \\
\hline N. E. $[\mathbf{v}]$ & mhm & & & \\
\hline U. $[\mathbf{v}]$ & acht, nicht? & Mein älterer Brü-, Bruder hat & sechsundsechzig Jahre & und meine \\
\hline K. $[\mathbf{k}]$ & & Sprecher setzt neu an. & langer s-Anlaut & \\
\hline
\end{tabular}

$[21]$

\begin{tabular}{lllll}
\hline & $\ldots$ & $55[04: 42.8]$ & $56[04: 43.1]$ & $57[04: 45.0]$ \\
\hline A. B. $[\mathbf{v}]$ & jingste schwesda hod zwoaravierzg johr & $($.$) und samma no alle lema no alle$ & $(--)$ und \\
\hline N. E. $[\mathbf{v}]$ & \multicolumn{2}{c}{ mhm } & & \\
\cline { 2 - 5 } Ü. $[\mathbf{v}]$ & Schwester hat zweiundvierzig Jahre. & Und sind wir noch alle, leben wir noch alle. & Und alle \\
\hline
\end{tabular}

\begin{tabular}{lllll}
\hline & -. & $58[04: 47.6]$ & $59[04: 49.4]$ & $60[04: 51.3]$ \\
\hline A. B. [v] & alle johr mach ma a alle johr ned & $(--)$ mia homa drä & $(--)$ drä johr wos ma & $(--)$ unser \\
\hline Ü. $[\mathbf{v}]$ & Jahre machen wir, alle Jahre nicht, & wir haben dre-, & drei Jahre, was wir & unser \\
\hline
\end{tabular}

\begin{tabular}{llll}
\hline & $\ldots$ & $61[04: 52.9]$ & $62[04: 54.8]$ \\
\hline A. B. $[\mathbf{v}]$ & fest & $(-)$ gmocht hamand a so hinter da familie & $(-)$ nahand kemma alle bria(da) \\
\hline Ü. $[\mathbf{v}]$ & Fest & gemacht haben so unter der Familie, & dann kommen alle Brüder. \\
\hline K. $[\mathbf{k}]$ & & & - da von briada kaum verständlich \\
\hline
\end{tabular}

\begin{tabular}{l|lll}
\hline & $\ldots$ & 63 [04:57.1] & 64 [04:57.4] 65 [04:57.9] \\
\hline A. B. $[\mathbf{v}]$ & i han sogar bildln mit & $(-)$ i kant enks zoing & \\
\hline N. E. $[\mathbf{v}]$ & \multicolumn{3}{c}{ ja (-) } \\
\hline A. W. $[\mathbf{v}]$ & & & de meng ma no seng \\
\hline U. $[\mathbf{v}]$ & Ich habe sogar Bilder mit. & Ich könnte euch es zeigen. & Die möchten wir noch sehen \\
\cline { 2 - 4 } K. $[\mathbf{k}]$ & & & \\
\hline
\end{tabular}


$[25]$

\begin{tabular}{|c|c|c|c|c|c|}
\hline & $66[04: 58.3]$ & $67[04: 59.4]$ & $68[04: 59.9]$ & $69[05: 00.1]$ & $71[05: 02.9]$ \\
\hline A. B. $[v]$ & & (.) $\mathrm{ja}$ & & $(1,6)$ i wird holn owa & i hols \\
\hline N. E. $[\mathbf{v}]$ & ja (.) de meng ma seng & & $\mathrm{mh}$ & & ja $(-)$ gern \\
\hline A. W. $[\mathbf{v}]$ & nachand & & & ja & \\
\hline Ü. [v] & nachher. & Ja. & & Ich werde holen. Aber & ich hole sie. \\
\hline Ü. [v] & Ja, die möchten wir sehen. & & & Ja. & \\
\hline
\end{tabular}

$[26]$

\begin{tabular}{llll}
\hline & $72[31: 13.4]$ & $73[31: 16.1]$ & $74[31: 17.9]$ \\
\hline A. $\mathbf{W} . \mathbf{~} \mathbf{v}]$ & Oans (.) oa frag hama na (-) und zwar & $(--)$ wias $(-)$ es dahoam sagts & $(-)$ zu \\
\hline U. $[\mathbf{v}]$ & Eins, eine Frage haben wir noch - und zwar: & Wie ihr daheim sagt & zu \\
\hline K. $[\mathbf{k}]$ & Lachen im Hintergrund & & \\
\hline
\end{tabular}

[27]

\begin{tabular}{lll}
\hline & $75[31: 18.0]$ & $76[31: 19.6]$ \\
\hline A. B. $[\mathbf{v}]$ & äh & $(---)$ mada irda micha pfinsta $($.$) fräta samsta und sunnta$ \\
\hline A. W. $[\mathbf{v}]$ & de wochadagsnam & \\
\hline Ü. $[\mathbf{v}]$ & die Wochentagsnamen? & Montag, Dienstag, Mittwoch, Donnerstag, Freitag, Samstag und Sonntag. \\
\hline
\end{tabular}

Bereits dieser kurze Ausschnitt zeigt sprachliche Besonderheiten, die konkret auf eine bairisch geprägte Minderheitensprache hinweisen, vor allem auf die nordmittelbairische Übergangszone, wie sie bis heute im Herkunftsgebiet der deutschböhmischen Siedler im östlichsten Bayern und angrenzenden Westböhmen greifbar ist.

\subsection{Lautliche Charakteristika}

Typisch für viele auf bairischen Varietäten basierende Minderheitensprachen sind der Erhalt bzw. die Weiterentwicklung der historischen Protodiphthonge ie, üe, uo, die in der Standardsprache und zahlreichen weiteren Varietäten des Deutschen rezent monophthongisch realisiert werden. Der ausgeprägte Mischcharakter des Deutschböhmischen zeigt sich dabei in der wortweise unterschiedlichen Weiterentwicklung dieser Protodiphthonge. So existieren neben der für mittelbairische Varietäten konstitutiven Realisierung von ie und $\ddot{u} e$ als /ie/und von uo als /ue/ auch die in der traditionellen dialektologischen Literatur als gestürzte Diphthonge bezeichneten nordbairischen /عI/ (für die Protodiphthonge $i e$ und $\ddot{u}$ ) und /rov/ (für historisches $u o$ ). Belege für den aus sprachgeschichtlicher Perspektive konservativen Erhalt bzw. in diphthongischer Form weiterentwickelter Phoneme stellen folgende Formen dar: 
(1) [bruede]

'Bruder'

(2) [briede $]$

'Brüder'

(3) $[\beta \varepsilon I]$

'wie'

(4) [brovm]

'Buben'

Wie in dieser kurzen Auflistung ersichtlich, kommen neben den mittelbairischen Diphthongen /up/ und /ie / auch die nordbairischen Varianten / unterschiedliche rezente Realisierung belegt den aus lautlicher Sicht deutlichen Mischcharakter der Varietät und rechtfertigt die Zuordnung der deutschböhmischen Minderheitensprache São Bentos zur nordmittelbairischen Varietätengruppe, die im Binnenraum bis heute vergleichbare lautliche Strukturen aufweist.

Weiterhin charakteristisch ist für diese Gruppe von Minderheitensprachen die Weiterentwicklung des Protodiphthongs ei zu /op/ oder/oI/. Auch hier zeigt sich erneut der nordmittelbairische Mischcharakter der Minderheitensprache, da neben mittelbairischen /op/ nordbairisches /oI/ belegbar ist:

[ßoevs]

'weiß' (1. und 3. Person Singular von wissen)

(6) [dsoIn]

'zeigen'

Auf ein relativ kleines Gebiet im Binnenraum weist die Monophthongierung ehemaliger Langvokale hin, die bis heute im bayerisch-tschechischen Grenzraum nachweisbar ist und auch in der Aufnahme mit dem Sprecher A. B. mehrmals auftaucht:

[dre:]

'drei'

(8) [hetsedo:gs]

'heutzutage'

Aus moderner binnenbairischer Perspektive ist bemerkenswert, dass die rezent als Kennlautung für große Teile des Bairischen zu betrachtende sogenannte $l$-Vokalisierung in der brasilianischen Variante nicht greifbar ist. Postvokalisch bleibt der Lateral durchgehend konsonantisch erhalten. Es ist davon auszugehen, dass zur Zeit der Auswanderung der Deutschböhmen nach Brasilien in der zweiten Hälfte des 19. Jahrhunderts die Vokalisierung des Liquids im Auswanderungsgebiet noch nicht etabliert war:

(9) [Jurl]

'Schule' 
(10) $[$ E:lten $]$

'Eltern'

(11) [ho:ltn]

'halten'

Ebenfalls typisch für bairischbasierte Minderheitensprachen ist die Hebung von $a$ in gewissen lautlichen Umgebungen ( $a$-Verdumpfung). Dieser Lautwandel ist auch für das Deutschböhmische in Brasilien anhand verschiedener Lexeme belegbar:

(12) [ho:d]

(er) 'hat'

(13) $\left[\mathrm{g} \int \mathrm{n}\right.$ o:ded $]$

'geschnattert' (im Sinne von 'gesprochen')

(14) [ho:ltn]

'halten'

\subsection{Lexikalische Charakteristika}

Im Bereich der Lexik zeigt die Minderheitensprache ebenfalls deutlich ihre Zugehörigkeit zur bairischen Varietätengruppe. Entsprechende Kennformen bzw. Kennwörter finden sich bereits in dem kurzen Transkript (zu den bairischen Kennwörtern in deutschböhmischen Minderheitensprachen siehe Wildfeuer 2017). Das ehemalige Dualpronomen enk 'euch' (mit pluralischer Bedeutung) taucht darüber hinaus mehrmals in den aufgezeichneten Gesprächen auf. Auch das Lexem Deandla 'Dirnlein, Mädchen (Pl.)' ist vor allem im Bairischen belegt. Dies gilt ebenso für die im Binnenbairischen heute meist ausgestorbenen Bezeichnungen Ne:l 'Ahne, Großvater' und Na:l 'Ahne, Großmutter' (nicht in obiger Transkriptionsauswahl abgedruckt) der Gewährsperson A. B. und ganz besonders für die heute nur mehr in konservativen Ausprägungen des Bairischen existierenden Wochentagsnamen Irda 'Ertag, Dienstag' und Pfinsta 'Pfinztag, Donnerstag', die ebenfalls im Interview mit A. B. erhoben werden konnten. Auffällig ist das inzwischen im Binnenraum fast gänzlich verschwundene hinter im Sinne von 'unter' (siehe hierzu Schmeller 1872/1877, Bd. 1: Sp. 1745).

\subsection{Sprachkontaktphänomene}

Neben den bairischen Spezifika zeigen sich einige Sprachkontaktphänomene, vor allem im Bereich der Lexik. Exemplarisch soll hier zuerst der Beleg Schneidossi der Gewährsperson A. B. (nicht im obigen Transkript) herausgestellt werden. Das Lexem stellt eine hybride Bildung aus bairisch Schnei 'Schnee' und portugiesisch doce 'Süßigkeit' dar und bezeichnet ein bei den Deutschböhmen in der Weihnachtszeit beliebtes Süßgebäck mit Puderzucker. 
Der Sprecher A. B. verwendete zudem das portugiesische Lexem prefeito anstelle einer auf Bürgermeister basierenden deutschen Variante und thematisiert dies folgendermaßen:

(15) mia hama o durt des scho glernt von unserne Eltern, des is da prefeito, ne? Aber des is auf Portugiesisch prefeito. [...] Aber mia hama des scho a so glernt und hamma des mid dem richtinga Nam vo wos a prefeito is nimmer glernt

(Wir haben ja dort das schon gelernt von unseren Eltern, das ist der prefeito, nicht? Aber das ist auf Portugiesisch prefeito. [...] Aber wir haben das schon so gelernt und haben das mit dem richtigen Namen von was ein prefeito ist nicht mehr gelernt)

Bemerkenswert ist darüber hinaus, dass sich in der vorliegenden Aufnahme keine Codeswitching-Phänomene zeigen. Dies weist darauf hin, dass die Gewährsperson A. B. über eine ausgeprägte Kompetenz in der Minderheitensprache verfügt und somit ein kompetenzgesteuertes Codeswitching (referential function nach Appel \& Muysken 1987: 118121) unterbleiben kann.

\section{Sprachpolitische und soziolinguistische Aspekte, sprachliche Vitalität}

Vor allem in Bezug auf die Varietäten der Hunsrücker scheint sich auf den Spracherhalt negativ auszuwirken, dass innerhalb der Sprechergruppe tendenziell eine abwertende Einstellung bezüglich der eigenen Varietät vorherrscht, vor allem im Vergleich zur deutschen Standardsprache. Pupp Spinassé (2016: 83 u. 85) konnte im Rahmen ihrer Untersuchung zum Hunsrückischen aufzeigen, dass die Sprechenden ihre eigene Varietät ,als schlechtes Deutsch“ bezeichnen. Die Autorin hält hierzu prägnant fest: „Als Varietät deutscher Herkunft wird das Hunsrückische im Allgemeinen ständig mit dem Standarddeutschen aus Deutschland verglichen. Was davon abweicht, wird für »Fehler « gehalten. Der Entwicklungsprozess der Sprache, ihre internen Regeln und ihre Systematizität in der Phonetik-Phonologie, in der Syntax sowie in der Wortbildung werden nicht berücksichtigt - und sie wird auf »falsches«, »schlechtes« Deutsch reduziert.“ (Pupp Spinassé 2016: 87).

Die Varietät der Hunsrücker hat somit - zumindest teilweise - einen eher negativen Status. $\mathrm{Zu}$ dieser Einschätzung gelangt auch Ammon (2015: 370). Allerdings ist das obige Zitat insofern einzuschränken, als Pupp Spinassé (2016: 96) im Rahmen ihrer Befragungen von Sprechern zu dem Ergebnis kommt, dass $88 \%$ der Befragten auf die Frage, welche Sprache sie am schönsten finden, mit „Hunsrückisch“ antworteten. Wenn auch im Vergleich zur Standardsprache das Hunsrückische als tendenziell minderwertig eingeschätzt wird, so hat die allergrößte Mehrheit der Sprecher durchaus ein positives Bild der eigenen Minderheitensprache. Es scheint hier ein generelles Problem dachloser Minderheitensprachen (zu denen wir tendenziell das Hunsrückische zählen) durchzuscheinen, indem die deutschbasierte Varietät nicht mehr durch die mediale und schulische Präsenz einer deutschen Standardsprache gestützt wird und in der Folge einen Prestigeverlust erleidet. Ähnliches ist auch aus anderen deutschsprachigen Siedlungen belegt. 
Hierzu kann exemplarisch auf die Untersuchung von Huffines (1988: 68) zum Pennsylvania German und auf Salmons (1986: 160) generell zum Deutschen in den USA hingewiesen werden.

Der Verlust einer standardnahen Varietät als Dachsprache hat seine Ursache im Nationalisierungsprozess Brasiliens unter Präsident Getúlio Vargas ab den 1930er Jahren, der auf eine portugiesische Einsprachigkeit abzielte (vgl. Pupp Spinassé 2016: 85). Der Schulunterricht in Deutsch wurde eingestellt, die Varietäten wurden somit zunehmend dachlos. Ammon (2015: 370) hält hierzu fest: „1938 (nach manchen Quellen schon 1937), bei Beginn der Politik des Estado Novo, die offiziell bis 1945 dauerte, wurden in ganz Brasilien - ca. 1.300 deutsche Privatschulen, 2.000 Vereine, 70 Zeitungen und Periodika, sowie jeglicher Deutschunterricht verboten [...].“

Für das Hunsrückische kommt erschwerend hinzu, dass seitdem kaum eine schriftsprachliche Tradition existiert. Erst durch das Projekt ESCRITHU wurden in den letzten Jahren Regeln zur Verschriftung zur Verfügung gestellt (siehe Pupp Spinasé 2016: 88). $\mathrm{Ob}$ dies zu einer Aufwertung des positiven Selbstbilds gegenüber der eigenen Minderheitensprache führen kann, werden zukünftige sprachbiografische und soziolinguistische Studien herausfinden müssen.

Deutlich positiv dürften sich - neben der oben kurz angesprochenen Entwicklung von ESCRITHU - didaktische Projekte auswirken, die die deutschbasierte Minderheitensprache für den Erwerb der Standardsprache nutzen. Pupp Spinassé (2016: 86) hat hierzu das Projekt Methodische Aspekte des Deutschunterrichts in multilingualen Kontexten Portugiesisch-Hunsrückisch (Ens-PH) entwickelt. Dabei soll neben der Nutzbarmachung der deutschbasierten Minderheitensprache auch die Einstellung zur eigenen Sprache positiv gestärkt werden. Dies geschieht unter anderem durch eine Fokussierung auf eigenständige Entwicklungs- und Entlehnungsprozesse im Hunsrückischen. Damit ist die Hoffnung verbunden, dass die Unterstützung einer positiven Einstellung gegenüber der eigenen Minderheitensprache dadurch erfolgt, dass das Hunsrückische auch als Brücke beim Deutschlernen fungieren kann. Das didaktische Projekt ist deutlich kontrastiv ausgerichtet und daher gut anschlussfähig an das seit einigen Jahren auch in Deutschland verbreitete Konzept der „Inneren Mehrsprachigkeit“ im Schulunterricht.

Spracherhaltend wirkt sich auf die Varietäten zudem aus, dass nach Ammon (2015: 374) bei den Hunsrückern immer noch eine Tendenz zur Endogamie besteht, sodass das Deutsche als Familiensprache zum Teil weiterexistiert. Außerdem bestehen bis heute dörfliche Sprachgemeinschaften in abgelegenen Gebieten (siehe Ammon 2015: 374375). Ammon (2015: 376) berichtet zudem von seiner Beobachtung, dass in einigen Rathäusern im Gebiet der Hunsrücker eine Kommunikation auf Deutsch möglich ist. Dagegen findet das Deutsche als Sprache der Liturgie kaum mehr Verwendung (siehe Ammon 2015: 376). In Bezug auf deutschsprachige Printmedien ist die Versorgung der Hunsrücker als sehr dürftig einzustufen. Eine eigene Tages- oder Wochenzeitung existiert nicht mehr. Die Deutsche Zeitung (mit Sitz in São Paulo) ist eine brasilienweite Monatszeitung. Dies stellt im Vergleich zu früher einen starken Schwund des Zugangs zu deutschsprachigen Printmedien dar. Zudem weist die jüngere Generation der Hunsrücker eine stark eingeschränkte Lesekompetenz in Bezug auf das Deutsche auf (siehe Ammon 2015: 377).

Ammon (2015: 378) erwähnt zwar die Zunahme kultureller Aktivitäten, beklagt jedoch gleichzeitig das Fehlen ,,an zugkräftigen kulturellen und sprachpflegerischen Verbänden.“ Zudem „fehlen prominente überregionale Meinungsführer, die den Zusammen- 
halt fördern könnten“. Auch besteht seiner Ansicht nach nur wenig Kontakt zu deutschsprachigen Ländern und eine wenig entwickelte Kontaktaufnahme durch Tourismus (Ammon 2015: 378-379).

Zusammenfassend kann zu den Hunsrückern festgehalten werden, dass einerseits eine noch relativ große Anzahl an Sprechern vorhanden ist und die Varietät teilweise noch einen wichtigen Status als mündliche Familiensprache innehat, andererseits die tendenzielle Dachlosigkeit - in Verbindung mit einem eher negativen Prestige des Dialekts im Vergleich zur Standardsprache - sich kontraproduktiv auf den Erhalt des Hunsrückischen auswirkt.

Ob bei den Deutschböhmen São Bentos ein mit den Hunsrückern vergleichbares, tendenziell negatives Selbstbild vorherrscht, wurde bisher nicht gezielt erforscht. Bei den von den Verfassern des vorliegenden Beitrags geführten Interviews tauchte nur an einer Stelle eine dahingehend interpretierbare Aussage auf. Auf die Frage, ob ihr deutschlernender Enkelsohn auch Bairisch lernt, antwortet die 2011 interviewte, deutschböhmische Sprecherin E. S. folgendermaßen:

\section{Na:, Bairisch moin $i$ is für eahm zu schlecht \\ 'Nein, Bairisch meine ich ist für ihn zu schlecht'}

Ähnlich wie das Hunsrückische ist das Deutschböhmische als tendenziell dachlose Varietät einzustufen. Auch hier ist der Zugang zu den Printmedien deutlich eingeschränkt (eine Ausnahme stellt eventuell die brasilianische Monatszeitung Deutsche Zeitung aus São Paulo dar). Eine gewisse Präsenz des Deutschen ist durch kulturelle Einrichtungen gegeben. So existiert in São Bento z. B. eine deutschböhmische Tanzgruppe. Weitere Aktivitäten mit deutschem Hintergrund, wie z. B. das jährlich stattfindende Schlachtfest und Bauernball, nennt die offizielle Internetseite.

Aus sprachpolitischer Perspektive und auch in Bezug auf den Erhalt oder Verlust der deutschbasierten Minderheitensprachen ist zudem das Konzept der Spracharchipele (siehe Steffen \& Altenhofen 2014) tragfähig. Dieses Konzept, das im Folgenden kurz skizziert wird, trifft sowohl auf die Hunsrücker Brasiliens als auch auf die Mennoniten Belizes zu. Für die Deutschböhmen São Bentos hat es unserer Ansicht nach jedoch keine Gültigkeit.

Ein Merkmal zahlreicher Minderheitensprachen, auch deutschbasierter in Mittel- und Südamerika, ist die Beobachtung, dass die Gemeinschaften nicht geschlossen nebeneinander siedeln, sondern sich über ein größeres Gebiet verstreuen und die jeweiligen Varietäten nicht durchgehend räumlich nebeneinander existieren. In Fortführung bzw. Erweiterung eines traditionellen Sprachinsel-Begriffs halten hierzu Steffen \& Altenhofen (2014: 39) für die Hunsrücker Brasiliens und die Mennoniten Belizes Folgendes fest: „Die Sprachgemeinschaften haben vielmehr den Charakter eines Archipels, also einer Vielzahl zusammenhängender Inseln, zwischen denen verschiedene Formen von Kommunikation stattfinden."

Als kohäsionsstiftend betrachten Steffen \& Altenhofen (2014: 45) z. B., dass Mitglieder verschiedener Mennonitensiedlungen in Belize sich gegenseitig besuchen, um Handel zu betreiben und dabei Plautdietsch sprechen. Dabei bleibt die Kommunikation nicht auf Belize beschränkt, sondern reicht bis nach Paraguay und in die USA (siehe Steffen \& Altenhofen 2014: 47). Auch für die Hunsrücker bestätigen sie Ähnliches, indem sie festhalten, dass ,durch die Migration und Ausdehnung der Hunsrückergruppe typi- 
sche Produkte und im Gefolge die Sprache sich ausdehnten und sozusagen einen TeutoRiogradenser Kulturraum über die brasilianischen Grenzen hinaus bis nach Misiones (Argentinien und Paraguay) entwickelten“ (Steffen \& Altenhofen 2014: 45). Es ist somit von möglicherweise intakten Verflechtungen auszugehen.

Bemerkenswert ist in diesem Kontext, dass die Kommunikation dabei nicht auf die Mündlichkeit beschränkt bleibt, sondern auch die Schriftlichkeit erfasste, die zwar meist in Standarddeutsch erfolgte, diese jedoch durchaus mit Varianten der Minderheitensprachen durchsetzt ist, wie Steffen \& Altenhofen (2014: 49-50 u. 53) anhand von Briefen feststellen konnten.

Dieses Konzept der „Distanzkommunikation“ (Steffen \& Altenhofen 2014: 48) greift für die Hunsrücker und Mennoniten, jedoch nicht für die Gruppe der Deutschböhmen. Ein Spracharchipel konnte sich aufgrund des sehr begrenzten Raums der Ansiedlung in und um São Bento nicht entwickeln.

Im Vergleich zu den Hunsrückern und den Deutschböhmen ist die Versorgung der Mennoniten mit Printmedien deutlich ausgebauter. Sie haben eigene Zeitungen bzw. Zeitschriften, z. B. in Belize Der Leserfreund. Darüber hinaus existieren panamerikanische Zeitschriften wie z. B. Das Blatt, Die Mennonitische Post und Gemeinde unter dem Kreuz des Südens (siehe Ammon 2015: 393). Auch ist in den meisten Siedlungen Zugang zu Radiosendern möglich (siehe Ammon 2015: 392). Zudem ist vielfach eine Überdachung durch eine standardnahe Varietät, das sogenannte „Mennonitenhochdeutsch“ (von den Sprechern auch als „Hüegdeutsch“ bezeichnet), vor allem im religiösen Kontext, gegeben (siehe Ammon 2015: 382 u. 388; Steffen 2006: XLI). Generell spielt das Deutsche bzw. eine Varietät davon eine bedeutende Rolle in der Religionsausübung (siehe hierzu Kap. 2.1.). Dies ist in Bezug auf den Spracherhalt ein nicht zu unterschätzender Faktor.

Kritisch für den Erhalt des Deutschen sieht Ammon (2015: 393) die „unterentwickelten Schulen, mit - im Vergleich zur Mehrheitsgesellschaft - deutlich kürzerer Schulpflicht [...]. Deutsch wird dann nur als Fach und für die religiöse Erziehung gebraucht".

Kontakt zu Deutschsprachigen außerhalb der Täufergruppe ist nach Aussage von Steffen (2006: 57-58) bewusst limitiert um ,äußere Einflüsse von der Gemeinschaft fernzuhalten". Auch die bei den Mennoniten vorherrschende Endogamie begrenzt den Einfluss von außen und ist als spracherhaltend zu bewerten.

Für den Erhalt der Sprache der Mennoniten ist nach Steffen \& Altenhofen (2014: 57) jedoch vor allem die Wirksamkeit des Spracharchipels relevant: „Dass sich das Hunsrückische und das Plautdietsche in Lateinamerika überhaupt bis ins 21. Jahrhundert hinein gehalten haben, liegt aber unseres Erachtens weniger an der Rückbindung ans sprachliche Mutterland, sondern mehr an den Verbindungen innerhalb des Archipels.“

Überträgt man die Stufen der Vitalitätsskala der UNESCO-Arbeitsgruppe zum Atlas of the World's Languages in Danger, ergibt sich nach unserer Ansicht folgende Verteilung: Für das Hunsrückisch in Brasilien ist Stufe 4 (unsafe) anzunehmen, zumindest in dörflichen Gemeinschaften, wo die Varietät tatsächlich noch Familiensprache ist: „Most but not all children or families of a particular community speak their language as their first language, but it may be restricted to specific social domains (such as at home where children interact with their parents and grandparents).“(UNESCO 2003: 7).

Das Deutschböhmische dürfte im Vergleich zum Hunsrückischen in seinem Bestand deutlich mehr gefährdet sein, was einerseits daran liegt, dass die Sprecherzahl nur einen Bruchteil der Zahlen des Hunsrückischen erreicht und die Weitergabe an die jüngste 
Generation fast gänzlich zum Erliegen kam. Wir gehen deshalb von einer Vitalität aus, die zwischen der Stufe 3 (definitively endangered) und Stufe 2 (severely endangered) einzuordnen ist:

Definitively endangered (3): The language is no longer being learned as the mother tongue by children in the home. The youngest speakers are thus of the parental generation. At this stage, parents may still speak their language to their children, but their children do not typically respond in the language.

Severely endangered (2): The language is spoken only by grandparents and older generations; while the parent generation may still understand the language, they typically do not speak it to their children. (UNESCO 2003: 8)

Die Plautdietsch sprechenden Mennoniten in Belize sehen wir in Bezug auf ihre sprachliche Vitalität auf Stufe 5 (stable yet threatened), die folgendermaßen definiert ist: „The language is spoken in most contexts by all generations with unbroken intergenerational transmission, yet multilingualism in the native language and one or more dominant language(s) has usurped certain important communication contexts." (UNESCO 2003: 7).

\section{Deutsch als Fremdsprache in Mittel- und Südamerika mit einem Fokus auf Brasilien}

Einen radikalen Einschnitt für den Gebrauch der deutschen Sprache im öffentlichen Kontext in Brasilien stellte, wie bereits unter Kap. 4. angeführt, die Politik des Estado Novo unter Präsident Getúlio Vargas (1930-1945) dar (Ammon 2015: 370). Bereits 1937 wurde das Portugiesische zur alleinigen Unterrichtssprache deklariert und 1942 wurde das Deutsche komplett aus dem öffentlichen Leben verbannt (siehe hierzu Kaufmann 2003: 30-31). Die von Vargas praktizierte Sprachenpolitik fokussierte mit Ausnahme des Portugiesischen alle Sprachen in Brasilien (siehe Ammon 2015: 370).

Seit 1961 ist Deutsch als Fremdsprache (DaF) an den Schulen wieder erlaubt, ,das alte Niveau deutschsprachiger Institutionen [wurde jedoch] bei weitem nie wieder erreicht“ (Ammon 2015: 370). Dass die „Nachwirkungen der Politik des Estado Novo“ (Kaufmann 2003: 32) bis heute greifbar sind, ist an der gegenwärtig praktizierten Fremdsprachenpolitik des brasilianischen Staates ersichtlich. Vom ersten bis zum achten Schuljahr (,primäre Schulphase“) ist lediglich Portugiesisch (mit Ausnahme der Sprachen der indigenen Minderheiten) als Schulsprache erlaubt, erst ab der fünften Klassenstufe wird „der Unterricht in mindestens einer modernen Fremdsprache [...] obligatorisch $[\ldots]^{\text {“ }}$ (Kaufmann 2003: 32). Prinzipiell wäre auch für die „sekundäre Schulphase“ vom neunten bis zum elften Schuljahr Unterricht in einer weiteren modernen Fremdsprache verpflichtend, sofern dies von der Schule finanziell realisiert werden kann (siehe Kaufmann 2003: 32). Die Entscheidung über die Fremdsprache obliegt zwar der jeweiligen Schule, jedoch fällt die Wahl in den meisten Fällen auf Englisch oder Spanisch (siehe Ammon 2015: 1038). In Rio Grande do Sul, Santa Catarina und Paraná wird in den deutschsprachigen Gebieten meist ab der fünften Klasse (bisweilen aber auch bereits zu einem früheren Zeitpunkt) bis zur achten Klasse Deutsch als Fremdsprache unterrichtet, von der neunten bis zur elften Klasse jedoch Englisch (siehe Kaufmann 2003: 32). 
Im hunsrückischen Siedlungsgebiet existieren derzeit circa 250 staatliche Schulen, Deutsch fungiert jedoch in keiner der Schulen als Unterrichtsprache, und im Unterricht wird auch keine Differenzierung zwischen Deutsch-als-Fremdsprache-Lernenden und Deutsch-als-Erstsprechenden (wenn auch in Form einer Varietät) vorgenommen (siehe Ammon 2015: 375 und das unter Kap. 4. angeführte Projekt von Pupp Spinassé 2016: 86).

Einen positiven Impuls für die Entwicklung von Deutsch als Fremdsprache hat sicherlich der Marktwert der deutschen Sprache durch die zahlreichen Niederlassungen von deutschen Firmen in Brasilien (siehe Ammon 2015: 370). Diesen Sachverhalt bestätigt auch Hess-Lüttich (2013: 17), indem er ausführt, dass an brasilianischen Schulen und auch Hochschulen basierend auf ,ökonomischer [und] nostalgischer Motivation“ ein zunehmendes Interesse an der deutschen Sprache und an der Germanistik generell zu verzeichnen ist. Ammon (2015: 1040), der die Zahl der DaF-Lernenden und -Studierenden für den Zeitraum von 1985 bis 2010 untersuchte, attestiert eine (gewisse) Stabilität. Der Brasilianische Deutschlehrerverband/Associação Brasileira de Associações de Professores de Alemão (ABraPA) bemüht sich ebenfalls um eine Förderung von DaF und Germanistik (siehe Ammon 2015: 1042). Zudem interessiert sich in den Regionen mit deutschsprachigen Minderheiten (trotz des Sprachwechsels zur Mehrheitssprache) die jüngere Generation für DaF (siehe Ammon 2015: 1038). Diese Beobachtung ist auch auf die Nachfahren der Deutschböhmen zu übertragen, wo zahlreiche Jüngere DaF erlernen.

Für die Ausbildung von Deutschlehrerinnen und -lehrern stehen (im Gebiet der Hunsrücker) immerhin fünf Universitäten zur Wahl, zwei in Porto Alegre, eine in São Leopoldo und zwei weitere (siehe Ammon 2015: 376). Germanistik als Studienfach existiert in Brasilien an insgesamt 16 Universitäten (siehe Ammon 2015: 1039). Darüber hinaus gibt es unter anderem auch sieben Goethe-Institute, ein Goethe-Zentrum, vier deutsche Auslandsschulen und zahlreiche PASCH-Schulen, so genannte Partnerschulen von deutschen Schulen (siehe Ammon 2015: 1041).

Nach Aussage von Pupp Spinassé (2014: 13) ist Deutsch an Schulen, Universitäten und auch in Sprachkursen ,eine der meistgelernten Sprachen in Brasilien [...]“.

\section{Ausblick}

Die in den vorausgehenden Kapiteln näher vorgestellten deutschsprachigen Gruppen in Mittel- und Südamerika stellen - wie bereits einleitend dargelegt - nur einen kleinen Ausschnitt der Deutschsprachigkeit in dieser Region dar. Zahlreiche weitere deutschsprachige Siedlungen und deren system- und soziolinguistischen Verhältnisse sind bisher wenig erforscht. Exemplarisch kann hier unter anderen auf deutschsprachige Gruppen in Argentinien, Brasilien, Chile, Peru und Venezuela verwiesen werden, die noch nicht ausführlich im Zentrum des Forschungsinteresses standen. So wissen wir z. B. noch eher wenig zur aktuellen sprachlichen Situation der Wolgadeutschen in der Provinz Buenos Aires (Argentinien), des Launa-Deutschen/Laguna-Deutschen in der Gegend um den Llanquihue-See (Chile), der Bukowina-Deutschen (hierunter vor allem Deutschböhmen) in Paraná (Brasilien) oder der Alemannisch sprechenden Siedler in Colonia Tovar (Venezuela). 


\section{Literatur}

Altenhofen, Cléo Vilson

1996 Hunsrückisch in Rio Grande do Sul: Ein Beitrag zur Beschreibung einer deutschbrasilianischen Dialektvarietät im Kontakt mit dem Portugiesischen. Stuttgart: Franz Steiner.

Altenhofen, Cléo Vilson

2016 Standard und Substandard bei den Hunsrückern in Brasilien: Variation und Dachsprachenwechsel des Deutschen im Kontakt mit dem Portugiesischen. In Alexandra N. Lenz (Hrsg.), $103-129$.

Altenhofen, Cléo Vilson \& Jaqueline Frey

2006 Das bresilionische Deitsch unn die deitsche Bresilioner: en Hunsrickisch Red fo die Sprocherechte. Contingentia: Zeitschrift der Deutschen Abteilung in Zusammenarbeit mit der PostGraduierung im Bereich Literatur- und Sprachwissenschaft der Bundesuniversität Porto Alegre (UFRGS 1). 39-50.

Ammon, Ulrich

2015 Die Stellung der deutschen Sprache in der Welt. Berlin \& München: De Gruyter.

Appel, René \& Pieter Muysken

1987 Language contact and bilingualism. London: Edward Arnold.

Blau, Josef

1958 Baiern in Brasilien: Chronik der im Jahre 1873 begonnenen Besiedlung von Sao Bento in Brasilien durch arme Leute aus dem Böhmerwald. Gräfelfing: Edmund Gans.

Eller-Wildfeuer, Nicole

2016 Bairischsprachige Siedlungen in den USA und in Brasilien - Aktuelle Lage, Sprechertypologie und mehrsprachige Konstellationen. In Alexandra N. Lenz (Hrsg.), 45-60.

Ethnologue 2016 = Lewis, M. Paul, Gary F. Simons \& Charles D. Fennig (Hrsg.)

2016 Ethnologue: Languages of the World, Nineteenth edition. Dallas \& Texas. URL: <https:// www.ethnologue.com/country/bz/languages>, letzter Zugriff: 30.08.2016.

EXMARaLDA

URL: <http://exmaralda.org/de/>, letzter Zugriff: 21.12.2017.

Grabarek, Józef

2013 Zur Geschichte der deutschen Sprache im 20. Jahrhundert. Frankfurt a. M.: Peter Lang.

Hess-Lüttich, Ernest W. B.

2013 Deutschsprachige Enklaven in Südamerika: Hunsrückisch in Brasilien und Wallisisch in Argentinien: Zur Sprachinselforschung in Rio Grande do Sul und San Jerónimo Norte. Zeitschrift für interkulturelle Germanistik 4(1). 11-27.

Huffines, Marion Lois

1988 Lexical Borrowing and Linguistic Convergence in Pennsylvania German. In Max Kade Center for German-American Studies (Hrsg.), Yearbook of German-American Studies (Volume 23), 59-71. Lawrence: The Society for German-American Studies.

Kaufmann, Göz

1997 Varietätendynamik in Sprachkontaktsituationen: Attitüden und Sprachverhalten rußlanddeutscher Mennoniten in Mexiko und den USA. Frankfurt a. M. u. a.: Peter Lang.

Kaufmann, Göz

2003 Deutsch und Germanistik in Brasilien. In Hans-Gert Roloff (Hrsg.), Jahrbuch für Internationale Germanistik (XXXV/1), 29-39. Frankfurt a. M.: Peter Lang.

Lenz, Alexandra N. (Hrsg.)

2016 German Abroad: Perspektiven der Variationslinguistik, Sprachkontakt- und Mehrsprachigkeitsforschung. Göttingen: Vienna University Press.

Pupp Spinassé, Karen

2014 Sprachenpolitische und didaktische Reflexionen über den Deutschunterricht in einem bilingualen Kontext Brasiliens. In Katharina Herzig, Sabine Pfleger, Karen Pupp Spinassé \& Sabrina Sadowski (Hrsg.), Transformationen: DaF-Didaktik in Lateinamerika: Impulse aus Forschung und Unterrichtspraxis, 13-29. Tübingen: Stauffenburg. 
Pupp Spinassé, Karen

2016 Das brasilianische Hunsrückische: soziolinguistische Aspekte einer durch Sprachkontakt geprägten Minderheitensprache. In Alexandra N. Lenz (Hrsg.), 81-102.

Salmons, Joseph C.

1986 But Hossiers Do Speak German: An Overview of German in Indiana. In Max Kade Center for German-American Studies (Hrsg.), Yearbook of German-American Studies (Volume 21),

Santa Catarina 155-166. Lawrence: The Society for German-American Studies.

URL: <http://turismo.sc.gov.br/en/cidade/sao-bento-do-sul/>, letzter Zugriff: 06.09.2016.

Schmeller, Johann Andreas

1872/1877 Bayerisches Wörterbuch (Bd. 1-2 in 4 Teilen). Nachdruck der von G. Karl Frommann bearbeiteten 2. Ausgabe. München: Oldenbourg.

Steffen, Joachim

2006 Vereinzelte Sprachinseln oder Archipel? Die Mennonitenkolonien in Belize im englisch-spanischen Sprachkontakt. Kiel: Westensee.

Steffen, Joachim \& Altenhofen, Cléo V.

2014 Spracharchipele des Deutschen in Amerika: Dynamik der Sprachvernetzung im mehrsprachigen Raum. Zeitschrift für Dialektologie und Linguistik 81(1). 34-60.

UNESCO. Ad hoc Expert Group on Endangered Languages

2003 Language Vitality and Endangerment. URL: <http://www.unesco.org/culture/ich/doc/src/ 00120-EN.pdf>, letzter Zugriff: 30.08.2016.

Wildfeuer, Alfred

2016 Globale Baiern - Zur Auswanderung aus West- und Südwestböhmen. In Max Sprenzinger (Hrsg.), Bairisch in der Welt: Beiträge zur gleichnamigen Tagung am 16. und 17. November 2011 an der Universität Regensburg, 120-151. Regensburg: edition vulpes.

Wildfeuer, Alfred

2017 Sprachenkontakt, Mehrsprachigkeit und Sprachverlust: Deutschböhmisch-bairische Minderheitensprachen in den USA und in Neuseeland. Berlin \& Boston: De Gruyter.

Ziegler, Arne

1996 Deutsche Sprache in Brasilien: Untersuchungen zum Sprachwandel und zum Sprachgebrauch der deutschstämmigen Brasilianer in Rio Grande do Sul. Essen: Blaue Eule.

Nicole Eller-Wildfeuer, Regensburg (Deutschland)

Alfred Wildfeuer, Augsburg (Deutschland)

\section{Deutsch als Minderheitensprache in Afrika}

1. Deutsch in Afrika

2. Die sprachlichen Repertoires der deutschsprachigen Community in Namibia
3. Deutsch als Fremdsprache

4. Literatur

\section{Deutsch in Afrika}

Die deutsche Sprache konzentriert sich in Afrika auf den Süden des Kontinents: Deutschsprachige Minderheiten gibt es in Namibia und in Südafrika. In beiden Ländern sind 